\title{
Initiation of nutrition in the intensive care unit and the reasons for its delay: a prospective audit
}

\author{
R. D. Abeles ${ }^{1}$, R. Pearse ${ }^{1}$ and J. Powell-Tuck ${ }^{2}$ \\ ${ }^{1}$ Intensive Care Unit, Royal London Hospital, Whitechapel, London E1 1 BB and ${ }^{2}$ Centre for Adult and Paediatric \\ Gastroenterology, Wingate Institute, Royal London Hospital, London E1 1BB
}

The need for nutritional support during critical illness is well established; however the timing of its initiation has been debated. Current evidence favours early initiation of both enteral nutrition $(\mathrm{EN})^{(1)}$ and parenteral nutrition (PN) ${ }^{(2)}$ feeding. The ACCEPT trial ${ }^{(3)}$ prospectively showed an association between improved outcome and early EN, in line with the Canadian Guidelines ${ }^{(4)}$. In light of this we audited nutrition practice in a teaching hospital intensive care unit (ICU).

A prospective audit of all patients admitted to the Royal London Hospital ICU over a 24-d period in August 2006 was carried out. Patients who were discharged, died or fed orally within $24 \mathrm{~h}$ were excluded. Mode of nutrition, time to initiation and reason for delay in delivery, were recorded. Due to the observational nature of the audit ethical approval was not required.

Of fifty-four patients admitted, thirty-four were included (median APACHE II 18 (14-21 IQR)). Thirty-three patients received EN and one received PN. Twenty-six $(77 \%)$ patients received nutrition within $24 \mathrm{~h}$. Six (18\%) patients received nutrition at $24-48 \mathrm{~h}$ : four due to medical decision and two due to technical difficulties. Two $(6 \%)$ patients received nutrition at $>48 \mathrm{~h}$ due to a delayed medical decision. Median delay initiating nutrition was $14 \mathrm{~h}$ (IQR 5-23). Delays resulted from medical decision making (53\%), admission to ICU (3\%), technical difficulties (5\%) and awaiting nasogastric-tube-position confirmation (31\%).

In most patients, nutrition commenced within the guideline period of $48 \mathrm{~h}(94.1 \%)$. Delay in decision making was the biggest contributor (six out of eight patients fed $>24 \mathrm{~h}$, whereas one patient had PN initiated within $9 \mathrm{~h}$ following prompt decision). Technical difficulties and tube placement comprised $36 \%$ of time delay, although this only affected two patients in delaying nutrition $>24 \mathrm{~h}$. Improvement in these two areas would help to optimise nutritional support in the ICU.

1. Heyland DK (1998) Critl Care Clin 14 (3), 423-440.

2. Simpson F \& Doig GS (2005) Intensive Care Med 31, 12-23.

3. Martin CM, Doig GS, Heyland DK, Morrison T \& Sibbald WJ; Southwestern Ontario Critical Care Research Network (2004) Can Medl Assoc J 170 (2), $197-204$.

4. Heyland DK, Dhaliwal R, Drover JW, Gramlich L, Dodek P; Canadian Critical Care Clinical Practice Guidelines Committee (2003) Journal of Parenteral and Enteral Nutrition 27 (5), 355-373. 